\title{
Enhanced chemo-responsiveness in patients with sleep apnoea and end-stage renal disease
}

\author{
J. Beecroft*, J. Duffin* ${ }^{\#}$, A. Pierratos ${ }^{\star}$, C.T. Chan*, P. McFarlane* and P.J. Hanly ${ }^{+}$
}

ABSTRACT: Although sleep apnoea is very common in patients with end-stage renal disease, the physiological mechanisms for this association have not yet been determined. The current authors hypothesised that altered respiratory chemo-responsiveness may play an important role.

In total, 58 patients receiving treatment with chronic dialysis were recruited for overnight polysomnography. A modified Read rebreathing technique, which is used to assess basal ventilation, ventilatory sensitivity and threshold, was completed before and after overnight polysomnography. Patients were divided into apnoeic $(n=38$; apnoea/hypopnoea index $(\mathrm{AHI})$ $35 \pm 22$ events $\left.\cdot h^{-1}\right)$ and nonapnoeic $\left(n=20 ;\right.$ AHI $3 \pm 3$ events $\left.\cdot h^{-1}\right)$ groups, with the presence of sleep apnoea defined as an $A H I>10$ events $\cdot h^{-1}$. While basal ventilation and the ventilatory recruitment threshold were similar between groups, ventilatory sensitivity during isoxic hypoxia (partial pressure of oxygen $\left.\left(\mathrm{PO}_{2}\right) 6.65 \mathrm{kPa}\right)$ and hyperoxia $\left(\mathrm{PO}_{2} 19.95 \mathrm{kPa}\right)$ was significantly greater in apnoeic patients. Overnight changes in chemoreflex responsiveness were similar between groups.

In conclusion, these data indicate that the responsiveness of both the central and peripheral chemoreflexes is augmented in patients with sleep apnoea and end-stage renal disease. Since increased ventilatory sensitivity to hypercapnia destabilises respiratory control, the current authors suggest this contributes to the pathogenesis of sleep apnoea in this patient population.

KEYWORDS: Chemoreflex, dialysis, kidney failure, respiratory control

leep apnoea has been reported in up to $50-70 \%$ of patients with end-stage renal disease (ESRD) [1], which is at least 10 times higher than the prevalence reported in the general population [2]. The pathogenesis of sleep apnoea in patients with ESRD remains unclear. Although sleep apnoea is not corrected by conventional haemodialysis or peritoneal dialysis [3-5], it has been reversed both by nocturnal haemodialysis and kidney transplantation [6-8], indicating that its pathophysiology is uniquely associated with the development of chronic renal failure. Previous investigators have observed features of both central and obstructive sleep apnoea in patients with ESRD [3-9], which suggests that its pathogenesis is related to both destabilisation of central respiratory control and upper airway occlusion.

During nonrapid eye movement (NREM) sleep, respiratory drive operates predominantly under chemoreflex control [10]. Changes in chemoreflex responsiveness can promote periodic breathing during sleep by destabilising central respiratory control [11]. The chemoreflex response depends on the ventilatory recruitment threshold, which is the level of carbon dioxide tension $\left(\mathrm{PCO}_{2}\right)$ at which ventilation begins to respond to $\mathrm{CO}_{2}$, as well as the sensitivity or slope of the ventilatory response above the threshold. Increased chemoreflex sensitivity can destabilise respiratory control by increasing the likelihood of lowering $\mathrm{PCO}_{2}$ below the "apnoeic threshold" [12]. Increased threshold can destabilise respiratory control by increasing plant gain [11]. Due to the metabolic hyperbola that models the control of $\mathrm{PCO}_{2}$ by pulmonary gas exchange, a high ventilatory threshold destabilises respiratory control as small increases in ventilation result in large decreases in $\mathrm{PCO}_{2}$, which increases the likelihood of ventilatory overshoot. Periodic breathing can be associated with upper airway occlusion during sleep, possibly because of disproportionately greater drive to the inspiratory pump muscles than the upper airway dilators $[13,14]$. More recently, ventilatory instability has been reported in patients with severe obstructive sleep apnoea (OSA) reflected by a high "loop gain" [15], suggesting a role for central destabilisation in the pathogenesis of OSA. Chemoreflex responsiveness may be

\section{AFFILIATIONS}

Depts of *Medicine, and

${ }^{\text {\#} A n a e s t h e s i a ~ \& ~ P h y s i o l o g y, ~}$ University of Toronto, and

-Dept of Medicine, Humber River Regional Hospital, Toronto, ON, and ${ }^{+}$Dept of Medicine, University of Calgary, Calgary, AB, Canada.

CORRESPONDENCE

P.J. Hanly

1421 Health Sciences Center

3330 Hospital Drive NW

Calgary

$A B$

T2N 4N1 Canada

Fax: 14032836151

E-mail: phanly@ucalgary.ca

Received:

June 272005

Accepted after revision:

February 142006 
altered by the metabolic changes that accompany renal failure, such as metabolic acidosis [16], and possibly by uraemia or the accumulation of so-called "middle molecules"; therefore, it is likely that it contributes to the development of central or OSA or both. While altered chemoreflex responsiveness has been described in patients with ESRD [17-19], the association between chemoreflex responsiveness and sleep apnoea in such patients has not been determined.

Chemoreflex responsiveness changes throughout the 24-h cycle among healthy subjects $[20,21]$ and recent data from the current authors' laboratory [22] suggest that chemoreflex responsiveness may change significantly overnight in patients with OSA, raising the possibility that changes in chemoreflex responsiveness are related to the intermittent hypoxaemia and/or sleep disruption that such patients experience. It is not known whether overnight changes in chemoreflex responsiveness are modified by the development of ESRD or the initiation of chronic haemodialysis or whether they differ between those who have sleep apnoea and those who do not.

The objectives of the present study were to measure chemoreflex responsiveness in patients with ESRD in a comprehensive and standardised manner, to assess whether it changes overnight and to determine whether responsiveness is altered in a manner that could contribute to the development of sleep apnoea. These questions were addressed by comparing measurements of chemoreflex responsiveness before and after overnight polysomnography in a large group of patients with ESRD. The measurements used included ventilatory sensitivity, which is a major determinant of respiratory control system stability, and the ventilatory recruitment threshold, which is a reflection of the acid-base status [23] and also determines plant gain. In addition, since the subjects were awake while these measurements were made, the sub-threshold ventilation was also measured. This basal ventilation or nonchemoreflex drive to breathe is sometimes called the wakefulness drive to breathe because it is absent during sleep [24]. Therefore, basal ventilation reflects the state of awareness or arousal [25].

\section{METHODS}

\section{Patient recruitment and polysomnography}

The study protocol was reviewed and approved by the research ethics board at St. Michael's Hospital (Toronto, ON, Canada), and all patients gave written informed consent to participate in the study. Patients receiving conventional haemodialysis (CHD) or peritoneal dialysis (PD) were recruited for overnight polysomnography, which was performed and scored by registered polysomnographic technologists according to published criteria [26] as previously described [6]. Patients receiving CHD (in-centre during the day for $4 \mathrm{~h}, 3$ days $\cdot$ week $^{-1}$ ) underwent polysomnography within $24 \mathrm{~h}$ of their last dialysis session. Patients receiving PD (continuous cycler-assisted PD nocturnally with three to five exchanges during sleep and/or three to five manual exchanges throughout the day with a single exchange overnight) continued dialysis during polysomnography. At the time of polysomnography, patients completed a sleep history questionnaire and Epworth Sleepiness Scale. A detailed history of each patient's renal failure was obtained, including the cause of renal failure, duration of dialysis treatment, dialysis schedule and medications. A venous blood sample (3-5 mL) was drawn from the lower arm pre- and post-polysomnography to determine electrolytes, blood urea nitrogen (BUN) and serum creatinine. Dialysis adequacy was assessed in patients receiving $\mathrm{CHD}$ using the per cent reduction in urea (PRU) per dialysis session (PRU $=$ (pre-BUN-post-BUN)/(preBUN) $\times 100$ ) [27] and dialyser coefficient representing dialysis adequacy, a measure of urea clearance, corrected for body size, was used for patients receiving PD since PRU cannot assess the adequacy of this modality [28].

\section{Chemoreflex responsiveness}

Chemoreflex responsiveness was measured using a modified [29-31] Read rebreathing technique [32]. The modifications were as follows. 1) Hyperventilation for $5 \mathrm{~min}$ at the beginning of the test. This modification facilitated sub- and suprathreshold measures of ventilation, enabling the response threshold to $\mathrm{CO}_{2}$ to be measured directly rather than by extrapolation. 2) Maintenance of isoxia throughout the test. This provided an estimation of the individual contributions of the central and peripheral chemoreflexes.

Measurements were obtained during isoxic hypoxia (partial pressure of oxygen $\left.\left(\mathrm{PO}_{2}\right) 6.65 \mathrm{kPa}\right)$ and isoxic hyperoxia $\left(\mathrm{PO}_{2}\right.$ $19.95 \mathrm{kPa})$, and changes in ventilation were measured as the level of $\mathrm{PCO}_{2}$ rose. The main test parameters obtained were: sub-threshold or basal ventilation $(V \mathrm{~Eb})$; ventilatory recruitment threshold for carbon dioxide $(\mathrm{T})$; and supra-threshold slope, termed ventilatory sensitivity ( $S$; fig. 1 ). When the test is performed during hypoxia, the ventilatory response reflects the activity of the central and peripheral chemoreflexes, but when it is performed during hyperoxia, the ventilatory response predominantly reflects the activity of the central chemoreflex as hyperoxia attenuates the activity of the peripheral chemoreceptors [31]. Since the contributions of the central and peripheral chemoreflexes are assumed to be additive, the sensitivity of the peripheral chemoreflex can be estimated by calculating the difference between the slopes of the ventilatory responses during hypoxic and hyperoxic conditions [33]. All measures of chemoreflex responsiveness were performed during wakefulness, immediately before and after overnight polysomnography. In addition, blood was drawn from the radial artery the morning after polysomnography to determine arterial blood gases. A complete description of the rebreathing collection and analysis protocol has been previously published [33].

\section{Analysis}

Mean data and SD were analysed using repeated measures of ANOVA, Pearson's correlation, multiple linear regression analysis and unpaired t-tests. Nominal data were analysed using Chi-squared analysis. All p-values $<0.05$ were considered statistically significant.

\section{RESULTS}

\section{Patient demographics}

In total, 58 patients (37 male, 21 female) aged 27-69 yrs were studied. Of these, 51 patients were receiving treatment with CHD and seven were treated with PD. Patients were divided into an apnoeic group and nonapnoeic group based on an apnoea/hypopnoea index (AHI) of $\geqslant 10$ events $\cdot h^{-1}$ during their overnight sleep study. Table 1 shows the patient demographics and dialysis data. Sleep apnoea was present in 


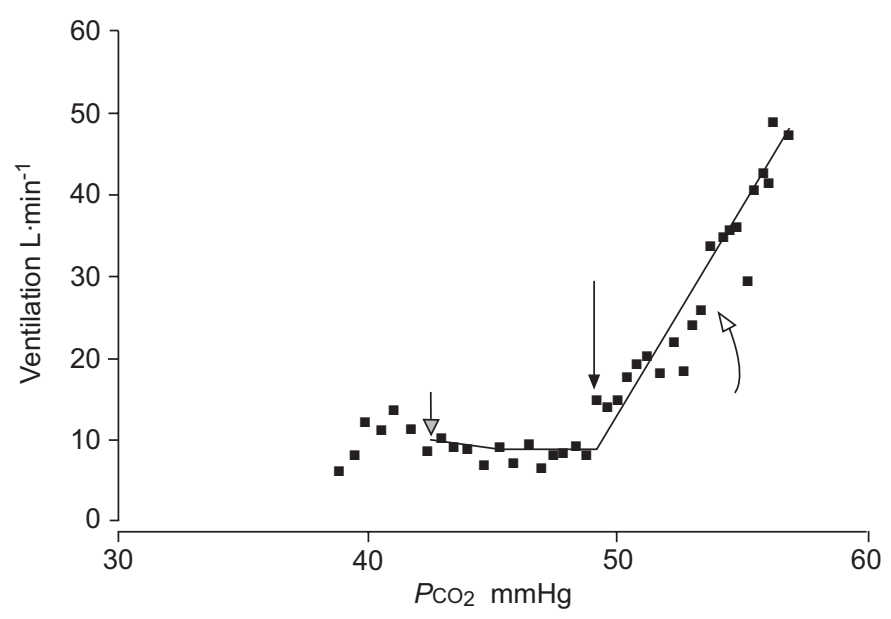

FIGURE 1. Example of a typical chemoreflex response. The main test parameters obtained were: sub-threshold or basal ventilation (grey arrow); ventilatory recruitment threshold for carbon dioxide (black arrow); and suprathreshold slope termed ventilatory sensitivity (open arrow). $\mathrm{PCO}_{2}$ : carbon dioxide tension. $1 \mathrm{mmHg}=0.133 \mathrm{kPa}$.

38 patients $(66 \%)$. Apnoeic patients were more likely to be male, older and have a higher body mass index (BMI). Measures of dialysis adequacy, BUN and serum creatinine did not differ between the two groups. There were no significant differences between groups in the proportion of patients taking cardiac or central nervous system medications.

\section{Polysomnographic and gas exchange data}

Polysomnographic data are shown in table 2 and the respiratory data for apnoeic patients are shown in table 3. By definition, AHI was significantly higher in the apnoeic group, apnoeas and hypopnoeas were predominantly obstructive $(89 \%)$, and a smaller proportion were classified as central $(7 \%)$ or mixed $(3 \%)$. In all patients, the majority $(>65 \%)$ of respiratory events had obstructive features. Mean arterial oxygen saturation $\left(\mathrm{Sa}_{1} \mathrm{O}_{2}\right)$ was lower and the percentage of total sleep time during which $\mathrm{Sa}_{1} \mathrm{O}_{2}$ was $<90 \%$ was higher in the apnoeic group. Mean transcutaneous carbon dioxide

\section{TABLE 1 Patient demographics and dialysis effectiveness}

\begin{tabular}{lccc} 
& Apnoeic & Nonapnoeic & p-value \\
\hline Patients $\mathbf{n}$ & 38 & 20 & \\
Male/female $\mathbf{n}$ & $28 / 10$ & $11 / 9$ & 0.031 \\
Age $\mathbf{y r s}$ & $50.7 \pm 11.3$ & $43.2 \pm 11.7$ & 0.020 \\
$\mathbf{B M I} \mathbf{~ k g} \cdot \mathbf{m}^{-\mathbf{2}}$ & $28.8 \pm 5.5$ & $25.0 \pm 4.2$ & 0.008 \\
PRU \% & $73.2 \pm 9.6$ & $71.4 \pm 10.7$ & NS \\
$\mathbf{~ K t} / \mathbf{V} \%$ & $2.33 \pm 0.49$ & 2.27 & \\
Creatinine $\boldsymbol{\mu} \mathbf{m o l} \cdot \mathbf{L}^{-1}$ & $687 \pm 238$ & $692 \pm 225$ & NS \\
Urea $\mathbf{m m o l} \cdot \mathbf{L}^{-1}$ & $16.2 \pm 6.6$ & $14.8 \pm 4.4$ & NS \\
\hline
\end{tabular}

Data are presented as $\mathrm{n}$ or mean $\pm \mathrm{SD}$. BMI: body mass index; PRU: per cent reduction in urea per dialysis session; Kt/N: dialyser coefficient representing dialysis adequacy; Ns: nonsignificant. \#: conventional haemodialysis only; ": peritoneal dialysis only. pressure $\left(P_{\mathrm{t}, \mathrm{CO} 2}\right)$ was not significantly different between groups. There were no inter-group differences in the arterial blood gas measurements, which were taken during wakefulness on the morning after the sleep study was completed (table 4).

Total sleep time and sleep efficiency did not differ between groups. Apnoeic patients had a significantly greater proportion of stage 1 NREM sleep and a smaller proportion of slow-wave sleep. There were no inter-group differences in other sleep stages or the frequency of periodic limb movements. The apnoeic group had a significantly greater number of arousals from sleep that were predominantly associated with apnoeas and hypopnoeas.

\section{Chemoreflex responsiveness}

The results of the rebreathing tests are shown in table 5. Within both groups, T was significantly lower while the $\mathrm{S}$ to $\mathrm{PCO}_{2}$ ratio was significantly higher during hypoxic as compared with hyperoxic rebreathing tests $(\mathrm{p}<0.001)$. The current authors also noted that the rate of rise of carbon dioxide $\left(\mathrm{rrCO}_{2}\right)$ in the rebreathing bag, which is a function of resting metabolic rate, was significantly higher during hypoxic as compared with hyperoxic conditions $(\mathrm{p}<0.001)$ along with measures of $V \mathrm{~Eb}$ $(p=0.008)$. There was a significant overnight decrease in the rate of $\mathrm{rrCO}_{2}$ and ventilatory recruitment threshold and a trend towards an overnight increase in ventilatory sensitivity, which did not reach statistical significance $(p=0.073)$. These changes were similar between apnoeic and nonapnoeic groups.

\begin{tabular}{|c|c|c|c|}
\hline \multirow[t]{2}{*}{ TABLE 2} & graphy & pnoeic an & apnoeic \\
\hline & Apnoeic & Nonapnoeic & p-value \\
\hline Total sleep time $\mathbf{h}$ & $5.2 \pm 1.2$ & $5.8 \pm 1.3$ & NS \\
\hline Sleep efficiency \% & $76.5 \pm 17.3$ & $83.9 \pm 16.6$ & NS \\
\hline Stage 1 NREM \% TST & $12.6 \pm 11.6$ & $6.1 \pm 3.9$ & 0.003 \\
\hline Stage 2 NREM \% TST & $53.7 \pm 14.6$ & $52.8 \pm 8.9$ & NS \\
\hline Slow-wave sleep \% TST & $16.2 \pm 9.7$ & $22.2 \pm 12.0$ & 0.043 \\
\hline REM \% TST & $17.4 \pm 8.5$ & $18.8 \pm 8.5$ & NS \\
\hline AHI events $\cdot h^{-1}$ & $35.3 \pm 21.5$ & $3.5 \pm 2.7$ & $<0.001$ \\
\hline Mean $\mathrm{Sa}, \mathrm{O}_{2} \%$ & $94.2 \pm 2.1$ & $95.5 \pm 1.9$ & 0.025 \\
\hline $\mathrm{Sa}, \mathrm{O}_{2}<\mathbf{9 0 \%} \%$ TST & $7.0 \pm 11.5$ & $2.1 \pm 5.3$ & 0.031 \\
\hline Mean $P_{\mathrm{t}, \mathrm{CO}_{2}} \mathbf{m m H g}$ & $45.0 \pm 4.4$ & $42.2 \pm 7.3$ & NS \\
\hline PLM events $\cdot h^{-1}$ & $19.5 \pm 27.0$ & $9.9 \pm 18.0$ & NS \\
\hline Total arousals events $\cdot h^{-1}$ & $42.3 \pm 22.9$ & $13.5 \pm 7.6$ & $<0.001$ \\
\hline $\begin{array}{l}\text { Respiratory arousals } \\
\text { events } \cdot h^{-1}\end{array}$ & $32.0 \pm 21.1$ & $2.9 \pm 2.3$ & $<0.001$ \\
\hline PLM arousals events $\cdot h^{-1}$ & $4.9 \pm 6.6$ & $2.8 \pm 6.4$ & NS \\
\hline $\begin{array}{l}\text { Spontaneous arousals } \\
\text { events } \cdot h^{-1}\end{array}$ & $5.2 \pm 4.1$ & $6.0 \pm 3.5$ & NS \\
\hline Awakenings events $\cdot h^{-1}$ & $7.0 \pm 8.0$ & $3.5 \pm 1.5$ & NS \\
\hline
\end{tabular}

Data are presented as mean \pm SD. NREM: nonrapid eye movement sleep; TST: total sleep time, expressed as a proportion of total study duration; REM: rapid eye movement sleep; $\mathrm{AHI}$ : apnoea/hypopnoea index; $\mathrm{Sa}, \mathrm{O}_{2}$ : arterial oxygen

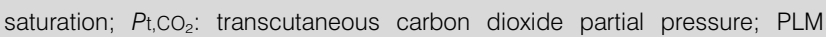
periodic limb movement; NS: nonsignificant. $1 \mathrm{mmHg}=0.133 \mathrm{kPa}$. 


\begin{tabular}{|c|c|c|c|c|c|}
\hline \multirow[t]{2}{*}{ TABLE 3} & \multicolumn{5}{|c|}{$\begin{array}{l}\text { Polysomnographic respiratory events in patients } \\
\text { with an apnoea/hypopnoea index }>10 \text { events } \cdot h^{-1}\end{array}$} \\
\hline & & Obstructive & Central & Mixed & Total \\
\hline \multicolumn{2}{|c|}{ Events per hour of sleep } & $30.4 \pm 16.7$ & $2.9 \pm 5.6$ & $1.9 \pm 5.2$ & $35.3 \pm 21.5$ \\
\hline \multicolumn{2}{|c|}{ Events during $R E M \cdot h^{-1}$} & $36.1 \pm 26.0$ & $1.0 \pm 3.9$ & $3.4 \pm 11.6$ & $40.5 \pm 24.7$ \\
\hline \multicolumn{2}{|c|}{ Events during NREM $\cdot h^{-1}$} & $28.7 \pm 17.9$ & $3.2 \pm 6.3$ & $1.9 \pm 4.9$ & $33.8 \pm 23.3$ \\
\hline \multicolumn{2}{|c|}{$\%$ of total events } & $89.6 \pm 15.1$ & $7.1 \pm 10.6$ & $3.3 \pm 8.0$ & \\
\hline
\end{tabular}

Data are presented as mean \pm SD. REM: rapid eye movement sleep; NREM: nonrapid eye movement sleep.

There were no inter-group differences in $V \mathrm{~Eb}$, ventilatory recruitment threshold or the $\mathrm{rCO}_{2}$. However, the ventilatory sensitivity to $\mathrm{PCO}_{2}$ was significantly higher in the apnoeic group for both hypoxic and hyperoxic rebreathing tests. In addition, the difference in ventilatory sensitivity between hypoxic and hyperoxic rebreathing tests was significantly greater in the apnoeic group. Figure 2 provides a graphical representation of these findings.

There was a significant positive correlation between hypoxic and hyperoxic ventilatory sensitivity and the severity of sleep apnoea, reflected by the AHI, and between hyperoxic ventilatory sensitivity and BMI (table 6). Further analysis of these variables by multiple linear regression identified AHI and excluded BMI as significant predictors of ventilatory sensitivity (evening hypoxic: model $\mathrm{r}^{2}=0.104$, AHI $\beta=0.322$, $\mathrm{p}<0.05$, BMI excluded; morning hypoxic: model $\mathrm{r}^{2}=0.205$, AHI $\beta=0.453, p<0.005$, BMI excluded; evening hyperoxic: model $\mathrm{r}^{2}=0.400$, AHI $\beta=0.545, \mathrm{p}<0.001$, BMI $\beta=0.193, \mathrm{p}=0.096$; morning hyperoxic: model $\mathrm{r}^{2}=0.275$, AHI $\beta=0.437, \mathrm{p}<0.005$, BMI $\beta=0.168, p=0.229$ ). There was no significant correlation between age and ventilatory sensitivity. Independent of apnoea designation, ventilatory sensitivity did not differ between sexes. In apnoeic patients, the change in ventilatory sensitivity from evening to morning did not correlate with AHI (hypoxic: $\mathrm{r}=0.156, \mathrm{p}=0.456$; hyperoxic $\mathrm{r}=-0.026, \mathrm{p}=0.893$ ). However, in nonapnoeic patients, the evening to morning change in hypoxic ventilatory sensitivity correlated with the AHI $(r=0.640, p=0.014)$, whereas the change in hyperoxic sensitivity did not $(r=-0.069, p=0.793)$.

\section{DISCUSSION}

A high prevalence of sleep apnoea was found in patients with ESRD, which is consistent with previous reports in this patient population [3-6, 9]. The majority of the apnoeas and hypopnoeas observed were obstructive events, which contrasts with many previous studies that reported a more heterogeneous apnoea profile consisting of central, mixed and obstructive events [4-6, 8]. The current study patients with sleep apnoea were heavier, older and were predominantly male, which may explain the predominance of obstructive events. However, these differences were small and neither BMI nor age were predictors of apnoea severity when included in regression analysis models. Nevertheless, these findings support the notion that sleep apnoea is common in patients with ESRD and that its pathogenesis is influenced by factors

\section{TABLE 4 Arterial blood gases}

\begin{tabular}{llll} 
& Apnoeic & Nonapnoeic & p-value \\
\hline $\mathbf{p H}$ & $7.40 \pm 0.04$ & $7.40 \pm 0.04$ & NS \\
$\mathbf{P C O}_{2} \mathbf{~ m m H g}$ & $41.3 \pm 3.7$ & $42.3 \pm 2.1$ & NS \\
$\mathbf{P O}_{2} \mathbf{~ m m H g}$ & $85.0 \pm 17.3$ & $93.8 \pm 15.1$ & NS \\
$\mathbf{H C O}_{3} \mathbf{~ m m o l} \cdot \mathbf{L}^{-1}$ & $26.7 \pm 3.3$ & $26.8 \pm 2.9$ & NS \\
$\mathbf{S a}_{\mathbf{0}} \mathbf{O}_{2} \%$ & $94.8 \pm 5.2$ & $96.6 \pm 1.1$ & NS \\
\hline
\end{tabular}

Data are presented as mean $\pm \mathrm{SD}$. $P \mathrm{CO}_{2}$ : carbon dioxide tension; $\mathrm{PO}_{2}$ : partial pressure of oxygen; $\mathrm{HCO}_{3}$ : bicarbonate; $\mathrm{Sa}_{\mathrm{O}_{2}}$ : arterial oxygen saturation; NS: nonsignificant. $1 \mathrm{mmHg}=0.133 \mathrm{kPa}$.

that are uniquely associated with ESRD over and above the mechanisms that cause sleep apnoea in the general population.

One of these potential mechanisms is altered respiratory chemoreflex responsiveness. During NREM sleep, respiratory drive operates predominantly under chemoreflex control [10]. Increased dependence on chemical control makes the system more vulnerable to changes in the level of arterial $\mathrm{PCO}_{2}$ in response to ventilatory stimuli. Increased chemoreflex threshold and/or sensitivity can lead to ventilatory instability and oscillations in respiratory output by increasing the likelihood of lowering $\mathrm{PCO}_{2}$ below the apnoeic threshold [12]. High ventilatory threshold destabilises respiratory control because a small increase in ventilation causes a large decrease in $\mathrm{PCO}_{2}$ [11]. High ventilatory sensitivity destabilises respiratory control by facilitating an increased ventilatory response to sufficient ventilatory stimuli [11]. In other words, increased

\begin{tabular}{|c|c|c|c|c|c|}
\hline \multirow{3}{*}{\multicolumn{2}{|c|}{ TABLE 5}} & $\begin{array}{l}\text { eflex resp } \\
\text { in apnoei } \\
\text { soxic hyp }\end{array}$ & $\begin{array}{l}\text { onsiveness } \\
\text { and none } \\
\text { xia and hy }\end{array}$ & $\begin{array}{l}\text { to arterial } \\
\text { pnoeic grc } \\
\text { peroxia }\end{array}$ & $\begin{array}{l}\mathrm{CO}_{2} \\
\text { ups }\end{array}$ \\
\hline & & \multicolumn{2}{|c|}{ Apnoeic } & \multicolumn{2}{|c|}{ Nonapnoeic } \\
\hline & & Evening & Morning & Evening & Morning \\
\hline \multicolumn{6}{|l|}{ Hypoxia } \\
\hline \multicolumn{2}{|c|}{$\mathrm{rrCO}_{2} \mathrm{mmHg} \cdot \mathrm{s}^{-1 \#}$} & $0.08 \pm 0.01$ & $0.07 \pm 0.01$ & $0.08 \pm 0.02$ & $0.07 \pm 0.01$ \\
\hline \multicolumn{2}{|c|}{ VEb $L \cdot \min ^{-1}$} & $13.0 \pm 4.7$ & $13.4 \pm 6.8$ & $12.5 \pm 9.1$ & $11.1 \pm 6.6$ \\
\hline \multicolumn{2}{|l|}{$\mathrm{T} \mathrm{mmHg}^{\circ}$} & $41.3 \pm 3.3$ & $40.0 \pm 3.0$ & $41.1 \pm 3.5$ & $40.3 \pm 3.6$ \\
\hline \multicolumn{2}{|c|}{$\mathrm{SL} \cdot \mathrm{min}^{-1} \cdot \mathrm{mmHg}^{-1+}$} & $6.0 \pm 3.9$ & $7.3 \pm 3.5$ & $3.3 \pm 3.4$ & $3.5 \pm 1.5$ \\
\hline \multicolumn{6}{|c|}{ Hyperoxia } \\
\hline \multicolumn{2}{|c|}{$\mathrm{rrCO}_{2} \mathrm{mmHg} \cdot \mathrm{s}^{-1 \#}$} & $0.07 \pm 0.01$ & $0.07 \pm 0.01$ & $0.07 \pm 0.01$ & $0.07 \pm 0.01$ \\
\hline \multicolumn{2}{|c|}{ VEb $L \cdot \min ^{-1}$} & $12.6 \pm 5.1$ & $12.0 \pm 4.2$ & $9.0 \pm 8.0$ & $9.3 \pm 6.6$ \\
\hline \multicolumn{2}{|l|}{$\mathrm{T}_{\mathrm{mmHg}}$} & $46.7 \pm 2.5$ & $45.8 \pm 3.3$ & $46.3 \pm 3.5$ & $45.3 \pm 3.3$ \\
\hline \multicolumn{2}{|c|}{$\mathrm{S} \mathrm{L} \cdot \mathrm{min}^{-1} \cdot \mathrm{mmHg}^{-1+}$} & $2.7 \pm 1.1$ & $3.2 \pm 1.3$ & $1.4 \pm 0.5$ & $1.7 \pm 0.8$ \\
\hline \multicolumn{6}{|c|}{ Hypo-hyperoxia } \\
\hline \multicolumn{2}{|c|}{$\mathrm{SL} \cdot \mathrm{min}^{-1} \cdot \mathrm{mmHg}^{-1 \S}$} & $3.3 \pm 3.4$ & $4.1 \pm 2.9$ & $1.8 \pm 1.2$ & $1.8 \pm 3.4$ \\
\hline
\end{tabular}

Data are presented as mean $\pm \mathrm{SD}$. $\mathrm{rrCO}_{2}$ : rate of rise of carbon dioxide during the modified Read rebreathing test; $V_{E b}$ : basal ventilation; $\mathrm{T}$ : ventilatory threshold; S: ventilatory sensitivity. ${ }^{\#}$ : evening versus morning, $p<0.001$; evening versus morning, $\mathrm{p}=0.006{ }^{+}{ }^{+}$: apnoeic versus nonapnoeic, $\mathrm{p}<0.001 ;{ }^{\S}$. apnoeic versus nonapnoeic, $\mathrm{p}=0.030 .1 \mathrm{mmHg}=0.133 \mathrm{kPa}$. 

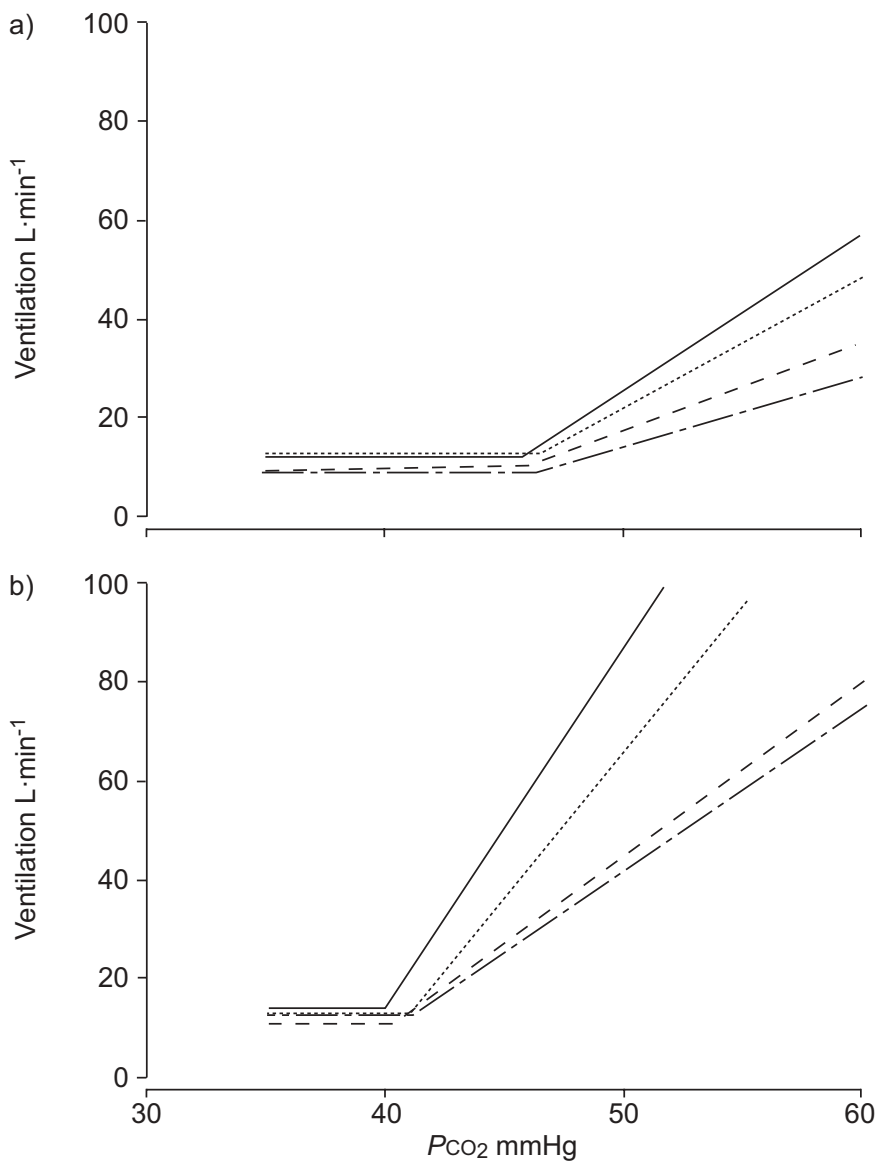

FIGURE 2. Mean chemoreflex responses in the apnoeic and nonapnoeic groups under a) hyperoxic and b) hypoxic conditions. Sub-threshold basal ventilation and ventilatory recruitment threshold are similar, whereas the suprathreshold slope (ventilatory sensitivity) is greater among apnoeic patients under both conditions. Ventilatory recruitment threshold decreases overnight in both groups under both conditions. $\mathrm{C}_{\mathrm{CO}_{2}}$ : carbon dioxide tension. $\cdots$ : apnoeic evening —: apnoeic morning; - - - : nonapnoeic evening; - - -: nonapnoeic morning. $1 \mathrm{mmHg}=0.133 \mathrm{kPa}$.

ventilatory threshold and/or sensitivity lead to ventilatory instability by increasing loop gain [11].

In the present study, apnoeic patients were found to have a higher ventilatory sensitivity to hypercapnia during both hypoxic and hyperoxic rebreathing tests. The ventilatory sensitivity also showed a significant positive correlation with apnoea severity. These data indicate that the sensitivity of both the central and peripheral chemoreceptors is increased in patients with sleep apnoea and ESRD, which the current authors anticipate destabilises respiratory control by increasing feedback and, therefore, loop gain [11]. Altered chemoreflex responsiveness has been well described in the pathogenesis of central sleep apnoea, including idiopathic central sleep apnoea [34], apnoea at high altitude [35] and Cheyne-Stokes respiration in patients with congestive heart failure [36]. Most of the apnoeas and hypopnoeas in the study patients were classified as obstructive events according to conventional polysomnographic criteria. However, there is abundant evidence that oscillation in respiratory control does

\begin{tabular}{|c|c|c|c|}
\hline & Age yrs & BMI $\mathbf{k g} \cdot \mathbf{m}^{-2}$ & $A H I$ events $\cdot h^{-1}$ \\
\hline \multicolumn{4}{|l|}{ Hypoxic S } \\
\hline \multicolumn{4}{|l|}{ Evening } \\
\hline r-value & 0.147 & 0.156 & $0.322^{*}$ \\
\hline $\mathrm{p}$-value & 0.329 & 0.301 & 0.029 \\
\hline \multicolumn{4}{|l|}{ Morning } \\
\hline r-value & 0.173 & 0.298 & $0.453^{*}$ \\
\hline $\mathrm{p}$-value & 0.285 & 0.062 & 0.003 \\
\hline \multicolumn{4}{|l|}{ Hyperoxic S } \\
\hline \multicolumn{4}{|l|}{ Evening } \\
\hline r-value & 0.059 & $0.363^{*}$ & $0.605^{\star \star \star}$ \\
\hline p-value & 0.673 & 0.007 & 0.000 \\
\hline \multicolumn{4}{|l|}{ Morning } \\
\hline r-value & 0.066 & $0.336^{\star}$ & $0.501^{\star \star \star}$ \\
\hline$p$-value & 0.654 & 0.019 & 0.000 \\
\hline
\end{tabular}

occur in patients with OSA. Periodic breathing was observed over 20 yrs ago following tracheostomy for the treatment of severe OSA [37, 38] and, more recently, ventilatory instability reflected by a high loop gain, has been reported in patients with severe OSA [15]. Furthermore, there is evidence that upper airway closure can occur during central sleep apnoea. Airway narrowing and closure was identified by bronchoscopic evaluation in healthy individuals with central apnoea that occurred spontaneously or was induced experimentally by transient hyperventilation [39]. Earlier studies attributed this phenomenon to disproportionate activation of the inspiratory muscles, which was greater or earlier than activation of the upper airway dilator muscles [14]. Others studies have reported that upper airway closure is more likely to occur during central apnoea in individuals whose upper airway is functionally or anatomically predisposed to collapse during sleep [40]. Consequently, the increased ventilatory sensitivity that was observed during wakefulness in the present study's patients may have led to ventilatory instability and obstructive apnoea during sleep.

The mechanisms responsible for increased ventilatory sensitivity among the apnoeic study patients are unclear. Chemoreflex responsiveness can be increased by metabolic acidosis; acetazolamide-induced metabolic acidosis enhances central chemosensitivity and reduces the ventilatory threshold to hypercapnia [41, 42]. However, the current blood gas data did not indicate acidosis, at least in the first $24 \mathrm{~h}$ after dialysis. Moreover, there was no difference in $\mathrm{pH}$ between apnoeic and nonapnoeic patients (table 4), nor $\mathrm{P}_{\mathrm{t}, \mathrm{CO}_{2}}$ measured during sleep (table 2). Furthermore, the ventilatory threshold to hypercapnia during isoxic hypoxia and hyperoxia did not differ between groups.

A single previous study reported that dialysis and uraemia may be associated with increased chemoreflex responsiveness [18]. It was suggested that removal of urea from the blood causes an influx of water into the brain where clearance of urea 
is slower, which may cause a "dilutional acidosis" at the central chemoreceptor, thereby increasing central chemoreflex sensitivity and threshold. However, no difference was found in either the severity of uraemia nor in the adequacy of dialysis in apnoeic patients compared with those without apnoea (table 1), suggesting that augmented chemoreflex responsiveness is not related to dialysis or uraemia in the study patients. In addition, there were no significant differences in chemoreflex responsiveness between patients on $\mathrm{CHD}$ and $\mathrm{PD}$, which suggests that increased ventilatory sensitivity is not due to changes in brain osmolality since PD does not lower urea like conventional haemodialysis does. The use of acetate buffer has been reported to augment apnoea severity in haemodialysis patients and has been attributed to alteration of ventilatory control [43]. However, all of the patients received bicarbonate buffer dialysis.

In the nonrenal failure population, OSA is associated with altered chemoreflex responsiveness [44]. This literature found increased ventilatory threshold and sub-threshold ventilation among apnoeic patients with no difference in ventilatory sensitivity, which differs from the current findings. While it is possible that the present findings were due to inherent differences between the apnoeic and nonapnoeic groups that were not related to renal failure, this seems unlikely, given the high prevalence of sleep apnoea in ESRD. It is also possible that increased ventilatory sensitivity is an adaptive response to the frequent bouts of hypoxia [45], hypercapnia and/or arousals associated with OSA. If this were true, greater overnight changes in chemoreflex measures in the apnoeic patients would have been anticipated, but this was not the case. Consequently, the current authors believe that the differences in chemoreflex responsiveness that were observed between apnoeics and nonapnoeics were due to the presence of renal failure. It is possible that increased ventilatory sensitivity is a consequence of factors that occur in chronic renal failure which were not measured in the present study, such as changes in autonomic tone [19] and/or the clearance of medium-sized molecules.

Significant evening to morning changes were also found in the chemoreflex responsiveness measurements. Specifically, the current authors found a decrease in the $\mathrm{rrCO}_{2}$ and ventilatory threshold overnight and a trend towards an overnight increase in ventilatory sensitivity that did not reach statistical significance, indicating an overnight increase in chemoreceptor activity. However, these changes were not specific to either the hypoxic or hyperoxic rebreathing test responses nor were they specific to apnoeic or nonapnoeic patients. Although the decline in the $\mathrm{rrCO}_{2}$ during the rebreathing tests and the overnight increase in ventilatory sensitivity would tend to destabilise respiratory control, these effects would be opposed by the overnight decrease in ventilatory threshold, which tends to stabilise the system. Previous reports from the current authors' laboratory in the nonrenal failure population [22] demonstrated an overnight decrease in ventilatory threshold that was confined to nonapnoeic patients and an overnight increase in ventilatory sensitivity that was confined to patients with OSA. The current findings are consistent with some previous studies', assessing the effect of circadian rhythm on chemoreflex control, which showed an evening to morning decline in metabolic rate [46] and an increase in chemoreflex sensitivity [20], but in contrast to others showing an evening to morning increase in ventilatory threshold [21]. It is not possible to determine how much of the overnight changes in chemoreflex responsiveness in the present study were due to renal failure, sleep apnoea or circadian influences, although the positive correlation $(\mathrm{r}=0.640, \mathrm{p}=0.014)$ between $\mathrm{AHI}$ and hypoxic ventilatory sensitivity in those with AHI $<10$ events $\cdot h^{-1}$ (nonapnoeic group) suggests that sleep apnoea may have some impact in patients with mild apnoea.

The study has some limitations that should be addressed. First, measurements of chemoreflex responsiveness were carried out during wakefulness and correlated with other respiratory measurements that were performed during sleep. The current authors acknowledge that the interaction between chemoreflex responsiveness and sleep onset plays a pivotal role in the development of sleep apnoea. However, it is believed that the manifestations of altered respiratory control observed during wakefulness continued during sleep and, therefore, contributed to the pathogenesis of sleep apnoea. Secondly, chemoreflex responsiveness varies significantly between individuals, which can make it difficult to find significant differences between groups of subjects. The fact that the present authors were able to find significant differences between apnoeics and nonapnoeics, despite this inherent variability in the measurement, makes the current findings more robust. Finally, the current study design was cross-sectional and consequently cannot infer causality between augmented chemoreflex responsiveness and sleep apnoea. Nevertheless, as outlined in the previous paragraphs, it is believed that the changes in chemoreflex responsiveness were not a consequence of OSA and that, more likely, they contributed to its pathogenesis.

In conclusion, sleep apnoea in end-stage renal disease is associated with an increase in respiratory chemoreflex sensitivity to hypercapnia during both hypoxic and hyperoxic conditions. These data indicate an increase in the sensitivity of both the central and peripheral chemoreflex responses. Increased chemoreflex sensitivity destabilises respiratory control, and so may contribute to the pathogenesis of sleep apnoea in end-stage renal disease. Further studies are required to confirm a causal relationship between increased chemoreflex sensitivity and obstructive sleep apnoea in this patient population, which the current authors anticipate will provide the basis for new therapeutic options.

\section{ACKNOWLEDGEMENTS}

The authors would like to thank J. Gabor and S. Mahammed for their technical assistance, and S. Crombach, J. Zaltzman and R. Prasad for their assistance with patient recruitment.

\section{REFERENCES}

1 Kraus MA, Hamburger RJ. Sleep apnea in renal failure. Adv Perit Dial 1997; 13: 88-92.

2 Young T, Palta M, Dempsey J, Skatrud J, Weber S, Badr S. The occurrence of sleep-disordered breathing among middle-aged adults. N Engl J Med 1993; 328: 1230-1235.

3 Wadhwa NK, Mendelson WB. A comparison of sleepdisordered respiration in ESRD patients receiving hemodialysis and peritoneal dialysis. Adv Perit Dial 1992; 8: 195-198. 
4 Mendelson WB, Wadhwa NK, Greenberg HE, Gujavarty K, Bergofsky E. Effects of hemodialysis on sleep apnea syndrome in end-stage renal disease. Clin Nephrol 1990; 33: 247-251.

5 Kimmel PL, Miller G, Mendelson WB. Sleep apnea syndrome in chronic renal disease. Am J Med 1989; 86: 308-314.

6 Hanly PJ, Pierratos A. Improvement of sleep apnea in patients with chronic renal failure who undergo nocturnal hemodialysis. N Engl J Med 2001; 344: 102-107.

7 Auckley DH, Schmidt-Nowara W, Brown LK. Reversal of sleep apnea hypopnea syndrome in end-stage renal disease after kidney transplantation. Am J Kid Dis 1999; 34: 739-744.

8 Langevin B, Fouque D, Leger P, Robert D. Sleep apnea syndrome and end-stage renal disease: cure after transplantation. Chest 1993; 103: 1330-1335.

9 Stepanski E, Faber M, Zorick F, Basner R, Roth T. Sleep disorders in patients on continuous ambulatory peritoneal dialysis. J Am Soc Nephrol 1995; 26: 751-756.

10 Skatrud JB, Dempsey JA. Interaction of sleep state and chemical stimuli in sustaining rhythmic ventilation. J Appl Physiol 1983; 55: 813-822.

11 Khoo MC. Determinants of ventilatory instability and variability. Respir Physiol 2000; 122: 167-182.

12 Dempsey JA, Smith CA, Przybylowski T, et al. The ventilatory responsiveness to $\mathrm{CO}_{2}$ below eupnoea as a determinant of ventilatory stability in sleep. J Physiol 2004; 560: 1-11.

13 Onal E, Burrows DL, Hart RH, Lopata M. Induction of periodic breathing during sleep causes upper airway obstruction in humans. J Appl Physiol 1986; 61: 1438-1443.

14 Hudgel DW, Chapman KR, Faulks C, Hendricks C. Changes in inspiratory muscle electrical activity and upper airway resistance during periodic breathing induced by hypoxia during sleep. Am Rev Respir Dis 1987; 135: 899-906.

15 Younes M, Ostrowski M, Thompson W, Leslie C, Shewchuk W. Chemical control stability in patients with obstructive sleep apnea. Am J Respir Crit Care Med 2001; 163: 1181-1190.

16 Oren A, Whipp BJ, Wasserman K. Effects of chronic acidbase changes on the rebreathing hypercapnic ventilatory response in man. Respiration 1991; 58: 181-185.

17 Burgess KR, Burgess EE, Whitelaw WA. Impaired ventilatory response to carbon dioxide in patients with chronic renal failure: implications for the intensive care unit. Crit Care Med 1994; 22: 413-419.

18 Hamilton RW, Epstein PE, Henderson LW, Edelman NH, Fishman AP. Control of breathing in uremia: ventilatory response to $\mathrm{CO}_{2}$ after hemodialysis. J Appl Physiol 1976; 41: 216-222.

19 Auinger M, Wanke T, Merkle M, et al. Effect of autonomic neuropathy on ventilatory response to progressive hypercapnia in dialysis patients. Nephrol Dial Transplant 1995; 10: 825-830.

20 Spengler CM, Czeisler CA, Shea SA. An endogenous circadian rhythm of respiratory control in humans. J Physiol 2000; 526: 683-694.
21 Stephenson R, Mohan RM, Duffin J, Jarsky TM. Circadian rhythms in the chemoreflex control of breathing. Am J Physiol Reg Int Comp Physiol 2000; 278: R282-R286.

22 Mahamed S, Hanly PJ, Gabor J, Beecroft J, Duffin J. Overnight changes in chemoreflex control in obstructive sleep apnoea patients. Respir Physiol Neurobiol 2005; 146: 279-290.

23 Duffin J. Role of acid-base balance in the chemoreflex control of breathing. J Appl Physiol 2005; 99: 2255-2265.

24 Fink BR. Influence of cerebral activity in wakefulness on regulation of breathing. J Appl Physiol 1961; 16: 15-20.

25 Shea SA. Behavioural and arousal-related influences on breathing in humans. Exp Physiol 1996; 81: 1-26.

26 Rechtschaffen A, Kales A. A manual of standardized terminology, techniques and scoring system for sleep stages of human subjects. Los Angeles, Brain Information Service/Brain Research Institute, 1968; pp. 1-60.

27 Jindal KK, Manuel A, Goldstein MB. Percent reduction in blood urea concentration during hemodialysis (PRU). A simple and accurate method to estimate Kt/V urea. ASAIO Trans 1987; 33: 286-288.

28 Churchill DN. Adequacy of peritoneal dialysis: how much dialysis do we need? Kidney Int Suppl 1994; 48: S2-S6.

29 Casey K, Duffin J, McAvoy GV. The effect of exercise on the central-chemoreceptor threshold in man. J Physiol 1987; 383: 9-18.

30 Duffin J, McAvoy GV. The peripheral-chemoreceptor threshold to carbon dioxide in man. J Physiol 1988; 406: 15-26.

31 Mohan R, Duffin J. The effect of hypoxia on the ventilatory response to carbon dioxide in man. Respir Physiol 1997; 108: 101-115.

32 Read DJC. A clinical method for assessing the ventilatory response to $\mathrm{CO}_{2}$. Austral Ann Med 1967; 16: 20-32.

33 Duffin J, Mohan RM, Vasiliou P, Stephenson R, Mahamed S. A model of the chemoreflex control of breathing in humans: model parameters measurement. Respir Physiol 2000; 120: 13-26.

34 Xie A, Rutherford R, Rankin F, Wong B, Bradley TD. Hypocapnia and increased ventilatory responsiveness in patients with idiopathic central sleep apnea. Am J Respir Crit Care Med 1995; 152: 1950-1955.

35 Lahiri S, Maret K, Sherpa MG. Dependence of high altitude sleep apnea on ventilatory sensitivity to hypoxia. Respir Physiol 1983; 52: 281-301.

36 Javaheri S. A mechanism of central sleep apnea in patients with heart failure. N Engl J Med 1999; 341: 949-954.

37 Onal E, Lopata M. Periodic breathing and the pathogenesis of occlusive sleep apnea. Am Rev Respir Dis 1982; 126: 676-680.

38 Weitzman ED, Kahn E, Pollack CP. Quantitative analysis of sleep and sleep apnea before and after tracheostomy in patients with the hypersomnia sleep apnea syndrome. Sleep 1980; 3: 407-423.

39 Badr MS, Toiber F, Skatrud JB, Dempsey J. Pharyngeal narrowing/occlusion during central sleep apnea. J Appl Physiol 1995; 78: 1806-1815.

40 Warner G, Skatrud JB, Dempsey JA. Effect of hypoxiainduced periodic breathing on upper airway obstruction during sleep. J Appl Physiol 1987; 62: 2201-2211. 
41 Vvok A, Duffin J, Kowalchuk JM, Paterson DH, Cunningham DA. Changes in chemoreflex characteristics following acute carbonic anhydrase inhibition in humans at rest. Exp Physiol 2000; 85: 847-856.

42 Swenson ER, Hughes JM. Effects of acute and chronic acetazolamide on resting ventilation and ventilatory responses in men. J Appl Physiol 1993; 74: 230-237.

43 Jean G, Piperno D, François B, Charra B. Sleep apnea incidence in maintenance hemodialysis patients: influence of dialysate buffer. Nephron 1995; 71: 138-142.
44 Mateika JH, Ellythy M. Chemoreflex control of ventilation is altered during wakefulness in humans with OSA. Respir Phsyiol Neurobiol 2003; 138: 45-57.

45 Mateika JH, Mendello C, Obeid D, Badr MS. Peripheral chemoreflex responsiveness is increased at elevated levels of carbon dioxide after episodic hypoxia in awake humans. J Appl Physiol 2004; 96: 1197-1205.

46 Mortola JP. Breathing around the clock: an overview of the circadian pattern of respiration. Eur J Appl Physiol 2004; 91: 119-129. 